\title{
A Literature Survey: Plant Leaf Diseases Detection Using Image Processing Techniques
}

\author{
K.Narsimha Reddy ${ }^{1}$, B.Polaiah ${ }^{2}$, N.Madhu ${ }^{3}$ \\ Assistant professor, Dept. of ECE, Vardhaman college of Engineering, telangana, India. \\ Research scholar, Department of ECE, KL University, Vaddeswaram, Guntur, Andhra Pradesh, India ${ }^{I}$. \\ Professor, Department of electronics and communication engineering, KL University, Vaddeswaram, \\ Vijayawada, Andhra Pradesh, India ${ }^{2}$ \\ Assistant Professor, Vardhaman college of engineering,Department of electronics and communication \\ Engineering, telangana, India ${ }^{3}$.
}

\begin{abstract}
This paper provides survey on different classification techniques that can be used for plant leaf diseases classification. Identification of symptoms of disease by naked eye is difficult for farmer. Crop protection in large frames is done by using computerized image processing technique that can detect diseased leaf using color information of leaves. There are so many classification techniques such as $k$-Nearest Neighbor Classifier, Probabilistic Neural Network, Genetic Algorithm, Support Vector Machine, and Principal Component Analysis, Artificial neural network, Fuzzy logic. Selecting a classification method is always a difficult task because the quality of result can vary for different input data. Plant leaf disease classifications have wide applications in various fields such as in biological research, in Agriculture etc. This paper provides an overview of different classification techniques used for plant leaf disease classification.
\end{abstract}

Keywords: K- means clustering, ANN, SVM, Neural network.

\section{Introduction}

Developing countries like India the economy is mainly depends on agriculture. Due to plant diseases the quality and quantity of agriculture product is reduced. Diseases to the plants caused mainly by the fungi and bacteria and the lifecycle of micro-organism is unable to predict. Some of the plant disease do not have visibility during early stage it only appears at that final stage. The purpose of agriculture is not only to feed ever growing population but it is an important source of energy and a solution to solve the problem of global warming. Plant disease diagnose is very important in earlier stage in order to cure and control the disease. In this method experts are involved who have the ability to detect the changes in leaf color. Many times different experts identify the same disease as the different disease. This method is expensive as it requires continuous monitoring of experts. Depending on the applications, many systems have been proposed to solve or at least to reduce the problems, by making use of image processing, pattern recognition and some automatic classification tools. In the next section this paper tries to present those proposed systems in meaningful way.

\section{Literature Survey}

In this section describes various approaches for detecting the disease in plant leaf using image processing technique

Sachin D. Khirade \& et al... [1] Identification of the plant diseases is the key to preventing the losses in the yield and quantity of the agricultural product. It requires tremendous amount of work, expertize in the plant diseases, and also require the excessive processing time. Hence, image processing is used for the detection of plant diseases. Disease detection involves the steps like image acquisition, image pre-processing, image segmentation, feature extraction and classification. This paper discussed the methods used for the detection of plant diseases using their leaves images. This paper discussed various techniques to segment the disease part of the plant. This paper also discussed some Feature extraction and classification techniques to extract the features of infected leaf and the classification of plant diseases. The accurately detection and classification of the plant disease is very important for the successful cultivation of crop and this can be done using image processing. This paper discussed various techniques to segment the disease part of the plant. This paper also discussed some Feature extraction and classification techniques to extract the features of infected leaf and the classification of plant diseases. The use of ANN methods for classification of disease in plants such as self- organizing feature map, back propagation algorithm, SVMs etc. can be efficiently used. From these methods, we can accurately identify and classify various plant diseases using image processing technique.

Prof. Sanjay, B. Dhaygude\& et al... [2]The application of texture statistics for detecting the plant leaf disease has been explained Firstly by color transformation structure RGB is converted into HSV space because HSV is a good color descriptor. Masking and removing of green pixels with pre-computed threshold level. Then in the 
next step segmentation is performed using 32 X32 patch size and obtained useful segments. These segments are used for texture analysis by color co-occurrence matrix. Finally if texture parameters are compared to texture parameters of normal leaf.

Amandeep Singh, Maninder Lal Singh\& et al... [3] The most significant challenge faced during the work was capturing the quality images with maximum detail of the leaf color. It is very typical task to get the image with all the details within a procesable memory. Such images are formed a through high resolution and thus are of 6-10MB of size. This was handled by using a Nikon made D5200 camera which served the task very well. Second challenge faced was to get rid of illumination conditions as from the start to the end of paddy crop season, illumination varies a lot even when the image acquiring time is fixed. However the solution to this is variable user defined thresholding and making necessary adjustments to the shades of LCC.

M.Malathi, K.Aruli \& et al... [4]They provides survey on plant leaf disease detection using image processing techniques. Disease in crops causes significant reduction in quantity and quality of the agricultural product. Identification of symptoms of disease by naked eye is difficult for farmer. Crop protection especially in large farms is done by using computerized image processing technique that can detect diseased leaf using color information of leaves.Depending on the applications, many image processing technique has been introduced to solve the problems by pattern recognition and some automatic classification tools. In the next section this papers present a survey of those proposed systems in meaningful way.There are many methods in automated or computer vision for disease detection and classification but still there is lack in this research topic. All the disease cannot be identified using single method.

Malvika Ranjan, Manasi Rajiv Weginwar\& et al... [5] Describes a diagnosis process that is mostly visual and requires precise judgment and also scientific methods. Image of diseased leaf is captured .As the result of segmentation Color HSV features are extracted. Artificial neural network (ANN) is then trained to distinguish the healthy and diseased samples. ANN classification performance is $80 \%$ better in accuracy.

Y.Sanjana, AshwathSivasamy\& et al... [6]In this it describes the uploaded pictures captured by the mobile phones are processed in the remote server and presented to an expert group for their opinion. Computer vision techniques are used for detection of affected spots from the image and their classification. A simple color difference based approach is followed for segmentation of the disease affected lesions. The system allows the expert to evaluate the analysis results and provide feedbacks to the famers through a notification to their mobile phones. The goal of this research is to develop an image recognition system that can recognize crop diseases. Image processing starts with the digitized color image of disease leaf. A method of mathematics morphology is used to segment these images. Then texture, shape and color features of color image of disease spot on leaf were extracted, and a classification method of membership function was used to discriminate between the three types of diseases.

Bhumika S.Prajapati, Vipul K.Dabhi\& et al... [7]In this detection and classification of cotton leaf disease using image processing and machine learning techniques was carried out. Also the survey on background removal and segmentation techniques was discussed. Through this survey, we concluded that for background removal color space conversion from RGB to HSV is useful. We also found that thresholding technique gives good result compared to other background removal techniques. We performed color segmentation by masking green pixels in the background removed image and then applying thresholding on the obtained masked image to get binary image. This is useful to extract accurate features of disease. We found that SVM gives good results, in terms of accuracy, for classification of diseases. There are five major steps in our proposed work, out of which three steps have been implemented: Image Acquisition, Image pre-processing, and Image segmentation.

P.Revathi, M.Hemalatha\& et al... [8] This proposed work is based on Image Edge detection Segmentation techniques in which, the captured images are processed for enrichment first. Then R, G, B color Feature image segmentation is carried out to get target regions (disease spots). Later, image features such as boundary, shape, color and texture are extracted for the disease spots to recognize diseases and control the pest recommendation. In this Research work consist three parts of the cotton leaf spot, cotton leaf color segmentation, Edge detection based Image segmentation, analysis and classification of disease.

Mr. Pramod S. landge, Sushil A. Patil\& et al... [9]In this propose and experimentally evaluate a software solution for automatic detection and classification of plant diseases through Image Processing. Farmers in rural India have minimal access to agricultural experts, who can inspect crop images and render advice. Delayed expert responses to queries often reach farmers too late. This paper addresses this problem with the objective of developing image processing algorithms that can recognize problems in crops from images, based on colour, texture and shape to automatically detect diseases or other conditions that might affect crops and give the fast and accurate solutions to the farmer with the help of SMS. The design and implementation of these technologies will greatly aid in selective chemical application, reducing costs and thus leading to improved productivity, as well as improved produce.

Heeb Al Bashish, Malik Braik \& et al... [10]In this paper an image-processing-based approach is proposed and used for leaf and stem disease detection. We test our program on five diseases which effect on the plants; they are: Early scorch, Cottony mold, ashen mold, late scorch, tiny whiteness. The proposed approach is image- 
processing-based. In the first step of the proposed approach, the images at hand are segmented using the KMeans technique, in the second step the segmented images are passed through a pre-trained neural network. As a testbed we use a set of leaf images taken from Al-Ghor area in Jordan.

Satish Madhgoria, MarekSchikora\& et al... [11]Proposed automatic pixel based classification method for detecting unhealthy regions in leaf images is presented. The algorithms have been tested extensively. Linear SVM has been used to classify each pixel. We have also shown hoe the results from SVM could be improved remarkably using the neighborhood check technique. The presented algorithm could well extended for other detection tasks which also mainly rely on color information, but extension to other features is easily possible. The task is performed in three steps. First, we perform segmentation to divide the image into foreground and background. In the second step, support vector machines are applied to predict the class of each pixel belonging to the foreground. And finally, we do further refinement by neighborhood-check to omit all falsely-classified pixels from second step.

MarekSchikora, BalramNeupane \& et al...

[12]Thisalgorithmisdesignedtoattributeimagepixelsintooneofthetwoclasses: healthy andunhealthy. The taskissolvedinthreesteps.First,weperformsegmentationtodividetheimageintoforegroundandbackground. In thesecondstep,asupportvectormachine (SVM)isappliedto predict theclassofeachpixelbelongingtothe foreground.Andfinally, wedorefinementbyaneighborhood-checkin ordertoomitallfalselyclassifiedpixelsfrom thesecondstep.

\section{Conclusion}

This paper presents a survey on different method for plant leaf disease detection using image processing technique. There are many methods in automated or computer vision for disease detection and classification but still there is lack in this research topic. All the disease cannot be identified using single method. From study of above classification techniques we come up with following conclusion. The k-nearestneighbor method is perhaps the simplest of all algorithms for predicting the class of a test example. An obvious disadvantage of the k-NN method is the time complexity of making predictions. Additionally, neural networks are tolerant to noisy inputs. But in neural network it's difficult to understand structure of algorithm. SVM was found competitive with the best available machine learning algorithms in classifying high-dimensional data sets.

[1] Sachin D. Khirade, A.B Patil, "Plant Disease Detection Using Image Processing", International Conference on Computing Communication Control and Automation”, 2015.

[2] Prof. Sanjay B. Dhaygude, Mr.Nitin P.Kumbhar, "Agricultural plant Leaf Disease Detection Using Image Processing”, International Journal of Advanced Research in Electrical, Electronics and Instrumentation Engineering Vol. 2, Issue 1, January 2013.

[3] Amandeep Singh ,Maninder Lal Singh, "Automated Color Prediction of Paddy Crop Leaf using Image Processing”, International Conference on Technological Innovations in ICT for Agriculture and Rural Development (TIAR 2015), 2015.

[4] M.Malathi, K.Aruli , S.Mohamed Nizar, A.Sagaya Selvaraj, "A Survey on Plant Leaf Disease Detection Using Image Processing Techniques",International Research Journal of Engineering and Technology (IRJET),Volume: 02 Issue: 09, Dec 2015.

[5] Malvika Ranjan, Manasi Rajiv Weginwar, NehaJoshi, Prof.A.B. Ingole, "detection and classification of leaf disease using artificial neural network", International Journal of Technical Research and Applications, 2015.

[6] Y.Sanjana, AshwathSivasamy, SriJayanth, "Plant Disease Detection Using Image Processing Techniques",International Journal of Innovative Research in Science, Engineering and Technology, Vol. 4, Special Issue 6, May 2015.

[7] Bhumika S.Prajapati, Vipul K.Dabhi Harshadkumar, B.Prajapati, "A Survey on Detection and Classification of Cotton Leaf Diseases", International Conference on Electrical, Electronics, and Optimization Techniques (ICEEOT) - 2016.

[8] P.Revathi, M.Hemalatha, "Advance Computing Enrichment Evaluation of Cotton Leaf Spot Disease Detection U sing Image Edge detection", ICCCNT'12.

[9] Mr. Pramod S. landge, Sushil A. Patil, Dhanashree S. Khot, "Automatic Detection and Classification of Plant Disease through Image Processing”, International Journal of Advanced Research in Computer Science and Software Engineering, Volume 3, Issue 7, 2013.

[10] Heeb Al Bashish, Malik Braik, and Sulieman Bani-Ahmad, "A Framework for Detection and Classification of Plant Leaf and Stem Diseases", IEEE 2010

[11] Satish Madhgoria, MarekSchikora, and Wolfgang Koch, "Pixel-BasedClassificationMethodforDetectingUnhealthy RegionsinLeafImages", 2009.

[12] MarekSchikora, BalramNeupane, SatishMadhogaria, Wolfgang Koch, "Animageclassificationapproachtoanalyze thesuppressionofplant immunity bythe humanpathogenSalmonellaTyphimurium”, BMCBioinformatics2012. 